\title{
Pattern Formation in Consumer-Finite Resource Reaction-Diffusion Systems ${ }^{\dagger}$
}

By

\author{
Masayasu MimurA*
}

\section{$\S 1$. Introduction}

In 1952, two paradoxes of diffusion were demonstrated by simple differential equations. The first paradox was shown by a great mathematician, A. Turing, who is well known as a pioneer in the field of computer science. He proposed a simple reaction-diffusion (RD) system for which a spatially constant equilibrium state is possibly destabilized so that non-constant equilibrium states appear [1]. The occurrence of such destabilization indicates that diffusion does not necessarily enhance homogeneity in space. This instability is called "diffusion-induced instability". Mathematically speaking, it is some bifurcation phenomenon which is interpreted as the destabilization of spatially constant equilibrium solutions, when certain parameters in the system are suitably varied. He claimed that such destabilization plays a role in cell differentiation and morphogenesis arising in biological systems. The second was contributed by two neurophysiologists, A. L. Hodgkin and A. F. Huxley, who investigated the mechanism of impulses propagating along nerve fiber from experimental viewpoints [2]. One of the important neurophysiological problems was to understand the reason why a nerve impulse constantly propagates with fixed shape. In the same year as Turing' paradox was stated, they proposed a model of nonlinear partial differential equations to describe the propagation of impulses along fibers. The model is given by a coupling of a single RD equation with three ODEs. Since it contains high nonlinearity, its analysis was so hard at that time. But the model could be managed to numerically solve. It is thus

Communicated by H. Okamoto. Received April 27, 2004.

2000 Mathematics Subject Classification(s): 35K57, 92B05

${ }^{\dagger}$ This article is an invited contribution to a special issue of Publications of RIMS commemorating the fortieth anniversary of the founding of the Research Institute for Mathematical Sciences.

* Department of Mathematics, School of Science and Technology, Meiji University 
surprisingly revealed that it generates a localized wave which propagates with constant velocity. This should be another paradoxical evidence of diffusion, because their equations possibly generate a propagating localized wave, despite that they possess diffusion effect. Since two paradoxes were demonstrated in 1952 , RD systems of the form

$$
U_{t}=D \Delta U+F(U)
$$

have been intensively studied in the fields of not only mathematics but also applied sciences. Here $U=\left(u_{1}, u_{2}, \ldots, u_{n}\right), F(U)=\left(f_{1}(U), f_{2}(U), \ldots, f_{n}(U)\right)$ and $D$ is a diagonal matrix with positive elements $\left\{d_{i}\right\}(i=1,2, \ldots, n)$. Numerics has revealed that $\mathrm{RD}$ system of the form (1.1) generated so diverse complex patterns as well as waves arising in biology, chemistry, physics and other fields, in spite that it looks so simple. Let me show one simple but suggestive example of (1.1), which is the following two-component system for the unknowns $(u, v)$ :

$$
\left\{\begin{aligned}
\tau u_{t} & =\varepsilon \Delta u+\varepsilon^{-1} f(u, v) \\
v_{t} & =\Delta v+g(u, v)
\end{aligned}\right.
$$

with $f(u, v)=u\left(1-u^{2}\right)$ and $g(u, v)=u-u_{0}-\gamma\left(v-v_{0}\right)$ where $u_{0}=-\sqrt{2} / 2, v_{0}=$ $-\sqrt{2} / 4$, and $\tau, \varepsilon$ and $\gamma$ are positive constants. Suppose that the constant $\gamma$ is relatively large (satisfying $\gamma>1.74 \ldots$ ) so that the nulclines of $f$ and $g$ possess three intersecting points, say $\mathrm{P}, \mathrm{Q}$ and $\mathrm{R}$, as shown in Figure 1.

In the absence of diffusion, (1.2) reduces to the ODEs for the unknowns $(u(t), v(t))$

$$
\left\{\begin{aligned}
\tau \varepsilon u_{t} & =f(u, v) \\
v_{t} & =g(u, v)
\end{aligned}\right.
$$

which is called the Bohnhofer van der Pol equations [3]. By using phase plane analysis, one can easily find that the critical points $\mathrm{P}$ and $\mathrm{R}$ are both asymptotically stable, while Q is unstable, and moreover, for almost all initial data, the solution $(u(t), v(t))$ of $(1.3)$ converges to either $\mathrm{P}$ or $\mathrm{R}$. Therefore it turns out that (1.3) is a bistable system for which the dynamics of solutions is quite simple. Keeping this result in mind, let us go back to (1.2) in $\mathrm{R}^{\mathrm{N}}$ or in a bounded domain with zero-flux (Neumann) boundary conditions. Then we can also see that $\mathrm{P}$ and $\mathrm{R}$ are locally asymptotically stable, while $\mathrm{Q}$ is unstable, that is, (1.2) is a bistable RD system. Since a diffusion effect enhances spatial homogeneity, one may expect that the asymptotic behavior of solutions of (1.2) 


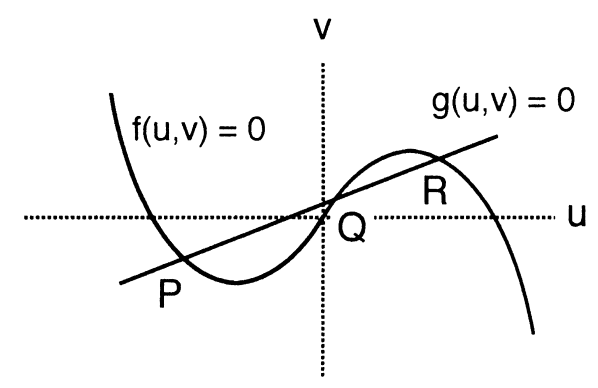

Figure 1. Nulclines of $f$ and $g$ in (1.2)

may inherit that of (1.3). Therefore, the following question naturally arises: "Is this expectation correct or not?". The limiting case when $\gamma \rightarrow \infty$ in (1.2) can be answered. In this case, $v$ becomes zero so that (1.2) and (1.3) reduce to the following scalar equations for $u$ only, respectively:

$$
\tau \varepsilon u_{t}=\Delta u+f(u, 0)
$$

and

$$
\tau \varepsilon u_{t}=f(u, 0)
$$

If (1.4) is considered in any convex domain with the zero-flux boundary condition, it is already known that any spatially non constant equilibrium solutions are unstable, even if they exist [4]. This implies that any solution of (1.4) is asymptotically described by the one of (1.5). That is, the expectation stated above is correct for very large $\gamma$. Then the next question is "How is the case when $\gamma$ is not very large?". Unfortunately, this question has not yet been analytically answered except for numerical computation. Let us show some numerical results on (1.2) with $\gamma=2.0$ where the nulclines of $f$ and $g$ are perfectly odd symmetric with its intersecting point $\mathrm{Q}$. We first show some remarks on the 1-dimensional traveling front solutions $(u, v)(z)(z=x-c t)$ with the velocity $c$ in $-\infty<z<\infty$, where the boundary conditions are

$$
(u, v)(-\infty)=\mathrm{P} \text { and }(u, v)(\infty)=\mathrm{R}
$$

[5] [6]. The first remark is that, due to the odd symmetry property of $f$ and $g$ $(\gamma=2.0)$, a standing (stationary) front solution, say SF, exists for any value of $\tau$, and that its stability depends on $\tau$. If $\tau$ is large, it is stable but when $\tau$ decreases, it destabilizes so that two traveling front solutions, say $\mathrm{TF}_{+}$with the 
velocity $c_{+}>0$ and $\mathrm{TF}_{-}$with $c_{-}<0$, super-critically and globally bifurcate from the trivial standing front one where $c_{+}=-c_{-}$, as in Figure 2. That is, if $\tau$ is chosen so as to be relatively small, then there exist two stable traveling front solutions (Figure 3) and one unstable standing front solution. Such multicoexistence of traveling front solutions is an essential feature in the system (1.2), because, the scalar equation (1.4) possesses a unique traveling front solution for any fixed value of $\tau$.

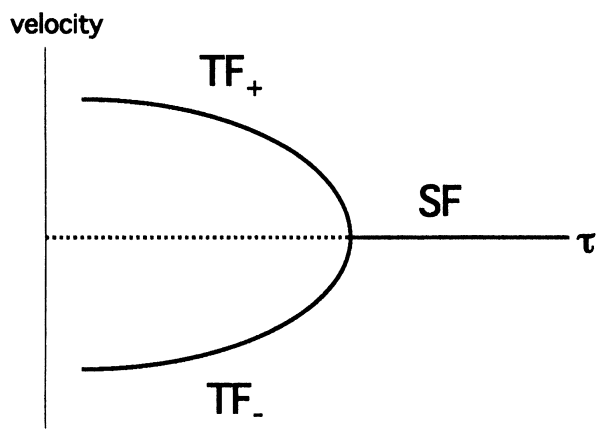

Figure 2. Pitchfork bifurcation

Keeping the information on traveling front solutions in mind, we consider (1.2) in a square domain $\mathrm{S}=\left\{(x, y) \in R^{2} \mid 0<x<L, 0<y<L\right\}$. Under the zero-flux boundary conditions, we compute (1.2) where $L=20, \varepsilon=0.05$, and $\gamma=3.5$ and $\tau$ is a free parameter. The first case is where $\tau=0.05$. Let the initial function $(u(0, x), v(0, x))$ be constructed in a way that it is approximated by $\mathrm{TF}_{+}$on the upper half domain of $\mathrm{S}$, and also approximated by $\mathrm{TF}_{-}$ on the lower half domain, as in Figure 4(a). Then for a short time, the resulting pattern twists to the clockwise direction, as in Figures 4(b), (c) and as time passes, rotating spiral pattern occurs, as in Figures 4(d), (e). This spatiotemporal pattern can not be expected by the information of the corresponding ODEs (1.3) only. This is certainly caused by the interplay of kinetics arising in ODEs and the diffusion effect in (1.2).

The second case is where $\tau=0.20$. The initial function is constructed similarly to Figure 4(a). For a short time, spiral pattern begins to form, as is expected. However, it breaks down and after large time, very complex irregular pattern appears, as in Figures $5(\mathrm{a}) \sim(\mathrm{c})$. Eventually, regular labyrinthine pattern is formed, as in Figures $5(\mathrm{~d})$, (e). These numerical results clearly indicate that RD systems of the form (1.2) possibly generate 

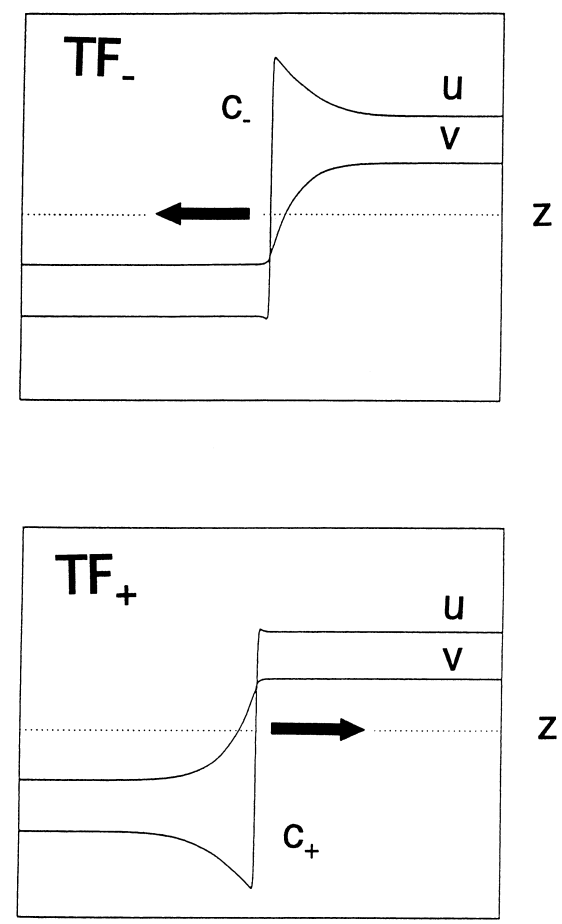

Figure 3. Traveling front solutions $\mathrm{TF}_{+}$and $\mathrm{TF}_{-}$

complex spatial and/or temporal patterns, which we are unable to expect from (1.3).

As is suggested by the above, we are interested in understanding what kind of patterns appear due to the interplay of reaction and diffusion. In this respect, the RD systems (1.2) in which any solution tends to be spatially homogeneous, in other words, no pattern appears, should be, one might say, discarded from our investigation, because they are less interesting from a pattern formation viewpoint.

We nevertheless would like to address the question: "Are RD systems of which any solution becomes spatially homogeneous really less interesting, from pattern formation viewpoints?" More precisely speaking, "Is the transient behavior of solutions also less interesting?". In this paper, we will introduce a class of consumer-finite resource RD systems and discuss this problem. Our purpose of the present paper is to say "No" to this question. 
(a)

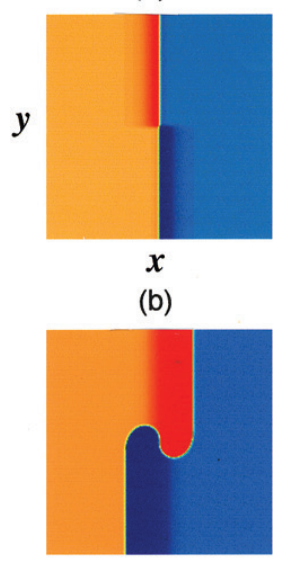

(c)

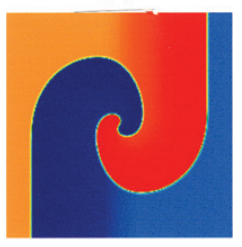

(d)

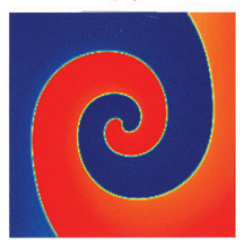

(e)

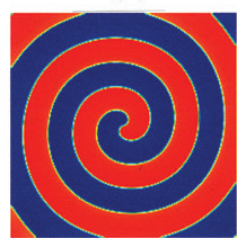

Figure 4. Formation of rotating spiral pattern 


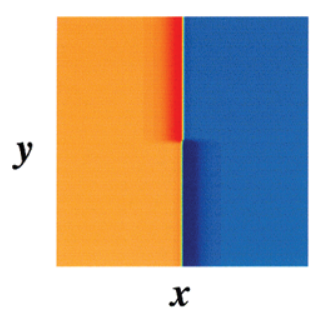

(a)

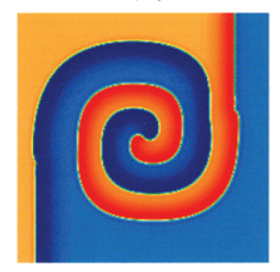

(b)

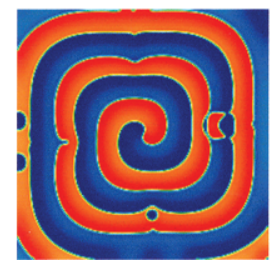

(c)

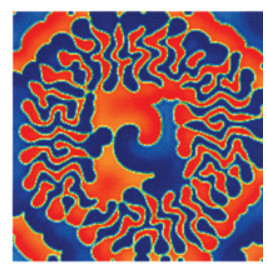

(d)

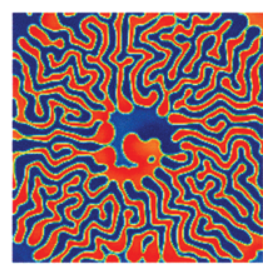

(e)

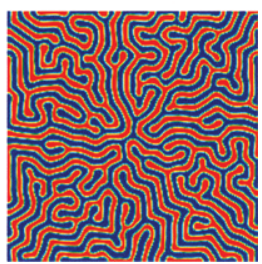

Figure 5. Formation of labyrinthine pattern

\section{§2. Consumer-Finite Resource RD Systems}

In this section, we restrict our discussion to the following class of RD systems for two components $(u, v)$ :

$$
\left\{\begin{array}{l}
u_{t}=d_{u} \Delta u+f(u) v-h(u) \\
v_{t}=d_{v} \Delta v-f(u) v
\end{array}\right.
$$

where $u$ and $v$ are called the consumer species and its resource, respectively. $d_{u}, d_{v}$ are the diffusion rates of $u$ and $v$, respectively. $f(u) v$ is the growth term where $f(u)$ is a increasing function satisfying $f(0)=0$ and $h(u)$ is the conversion (or dissipation) term where it is a non-decreasing function satisfying $h(0)=0$. Let us first introduce some examples described by $(2.1)$. 
Example 1. Epidemic diffusive model [7]

Let $u, v$ be respectively the infective and susceptible species which move by diffusion. The interaction between two species can be described by

$$
\left\{\begin{array}{l}
u_{t}=d_{u} \Delta u+k u v-a u \\
v_{t}=d_{v} \Delta v-k u v,
\end{array}\right.
$$

where $k$ is the contagion rate of the infective and susceptible species and $a$ is the removal rate of the susceptible species. Both are positive constants.

Example 2. Autocatalytic reactions [8]

Let us consider the following two chemical processes for three chemical substances $\mathrm{U}, \mathrm{V}$ and $\mathrm{P}$ :

$$
\begin{aligned}
\mathrm{V}+\mathrm{nU} & \rightarrow(\mathrm{n}+1) \mathrm{U} \\
\mathrm{mU} & \rightarrow \mathrm{P}
\end{aligned}
$$

When $k$ and $a$ are the reaction rates of (R1), (R2), respectively, the processes (R1), (R2) can be described by

$$
\left\{\begin{array}{l}
u_{t}=d_{u} \Delta u+k u^{n} v-a u^{m} \\
v_{t}=d_{v} \Delta v-k u^{n} v \\
p_{t}=a u^{m}
\end{array}\right.
$$

where $u, v$ and $p$ are the concentrations of the intermediate product $\mathrm{U}$, the reactant $\mathrm{V}$ and the final product $\mathrm{P}$. Here we assume that $\mathrm{P}$ is immobilized. $n$ and $m$ are some positive integers. It turns out that the first two equations are closed for $u$ and $v$ so that (2.3) falls into the framework of (2.1). In particular, for $n=2$ and $m=1,(2.3)$ is called the Gray-Scott systems without feeding process.

Example 3. Exothermic reaction-diffusion system [9]

A simplest exothermic reaction of some reactant $\mathrm{Z}$ is the following process:

$$
\mathrm{Z} \rightarrow \mathrm{P}
$$

where $\mathrm{P}$ is a product. Since the reaction is exothermic, the reaction rate depends on temperature, say $T$. It is given by $k(T)$, which is called the Arrehenius rate. Then the process is described by

$$
\left\{\begin{array}{l}
T_{t}=d_{T} \Delta T+\omega k(T) z-a T \\
z_{t}=d_{z} \Delta z-k(T) z
\end{array}\right.
$$


where $z$ is the concentration of the reactant $\mathrm{Z}, \omega$ is the conversion rate and $a$ is the rate of heat radiation. Both are positive constants. $k(T)$ is a increasing function of $T$.

For the system (2.1) in a bounded domain $\Omega$ (in $R^{N}$ ) with the zero-flux boundary conditions and the initial conditions

$$
(u, v)(0, x)=\left(u_{0}, v_{0}\right)(x) \geq 0 \quad x \in \Omega,
$$

asymptotic behavior of solutions $(u, v)$ are well studied. As an example of (2.1), we consider (2.3) and show some results.

Theorem 1 (Masuda [10]). Suppose $a=0$ in (2.3). Then for any positive integer $n$, there globally exists a nonnegative solution $(u, v)(t, x)$ satisfying

$$
\lim _{t \rightarrow \infty}(u, v)(t, x)=\left(<u_{0}+v_{0}>, 0\right)
$$

where $\langle s\rangle$ means the spatial average of $s(x)$ over $\Omega$.

Theorem 2 (Hoshino [11]). Suppose $a>0$. Then for any positive integer $n$ and $m$, there globally exists a nonnegative solution $(u, v)(t, x)$. Moreover,

(i) If $m$ and $n$ satisfy $0<m \leq n$, there exists some constant $v_{\infty}>0$ such that

$$
\lim _{t \rightarrow \infty}(u, v)(t, x)=\left(0, v_{\infty}\right)
$$

(ii) If $m$ and $n$ satisfy $0<n<m$,

$$
\lim _{t \rightarrow \infty}(u, v)(t, x)=(0,0) .
$$

These theorems imply that any solution of (2.3) asymptotically becomes spatially homogeneous and that its asymptotic behavior is qualitatively similar to the one of the ODEs corresponding to (2.3) in the absence of diffusion.

For a class of consumer-finite resource RD systems (2.1), it is intuitively expected that the consumer fades out after large time, that is, $\lim _{t \rightarrow \infty} u(t, x)=0$, because its resource is initially supplied, that is, finite. Therefore, one might conclude that the system (2.1) is less interesting from a pattern formation viewpoint. However, we have recently encountered some consumer-finite resource system arising in biology, which exhibits a very interesting transient behavior, despite that the asymptotic behavior is so trivial. It is a bacterial growth where the bacterium is a consumer, while the nutrient is its resource which is initially supplied. 


\section{§3. Bacterial Growth [12]}

Intensive experimental investigations have shown that the growth of bacterial colonies produces various complex patterns, depending on the species and environmental conditions. For instance, the bacterial species called Bacillus subtilis is known to exhibit many qualitatively different two-dimensional colony patterns as a result of growth and cell division on the surface of thin agar plates where nutrients are initially contained. It is observed that colony patterns change drastically when the initial concentrations of agar and nutrient, say $C_{a}$ and $C_{n}$, are globally varied. They are qualitatively classified into five types, each of which is observed in the regions labeled $\mathrm{A}-\mathrm{E}$ in the $\left(\mathrm{C}_{\mathrm{a}}\right.$, $1 / \mathrm{C}_{\mathrm{n}}$ )-parameter plane, as in Figure 6. In the region A (hard agar medium containing poor nutrients), colony patterns exhibit tip-splitting growth with characteristically branched structures (Figure 7). These patterns are similar to those observed in diffusion limited aggregation (DLA) processes in solidification from a supersaturated solution, solidification from an undercooled liquid, and electro-chemical deposition. Averages over about 25 samples of colony patterns in experiments yield a fractal dimension of $1.72 \pm 0.02$. This is indeed in good agreement with the results from two-dimensional DLA particle model. Increasing $C_{n}$ with a fixed value of $C_{a}$ large (corresponding to the change from the region $\mathrm{A}$ to $\mathrm{B}$ ), the branch thickness of the colony increases gradually and colony patterns eventually become Eden-like. For large $\mathrm{C}_{\mathrm{n}}$ and small $\mathrm{C}_{\mathrm{a}}$ (soft agar medium with rich nutrient) in the region $\mathrm{D}$, the corresponding colony pattern drastically changes to a homogeneously spreading one which looks macroscopically like a perfect disk. It is likely that the movement of bacterial cells inside a colony can be described in terms of diffusion, and there is no microscopic branching at all. In the region $\mathrm{E}$, between the regions $\mathrm{A}$ and $\mathrm{D}$, there emerge colony patterns clearly reminiscent of the so-called dense-branching morphology (DBM). Though the branching is very dense, the advancing envelope looks characteristically smooth compared with DLA-like colonies in the region A. Finally, in the region $\mathrm{C}$, between the regions $\mathrm{B}$ and $\mathrm{D}$, colonies spread and rest alternately, leaving stationary concentric ring-like patterns.

The bacterial growth system is certainly a consumer-finite resource system where the bacterial cells move by diffusion and the nutrients are diffusive in medium. Therefore, one could expect that the bacterial growth may be described by a kind of consumer-finite resource $\mathrm{RD}$ system. If so, any spatial pattern would not appear. Nevertheless, the experimental system clearly exhibits diverse complex patterns, depending on the environmental conditions. We therefore arrive at the following naïve question: "Is there any contradiction 


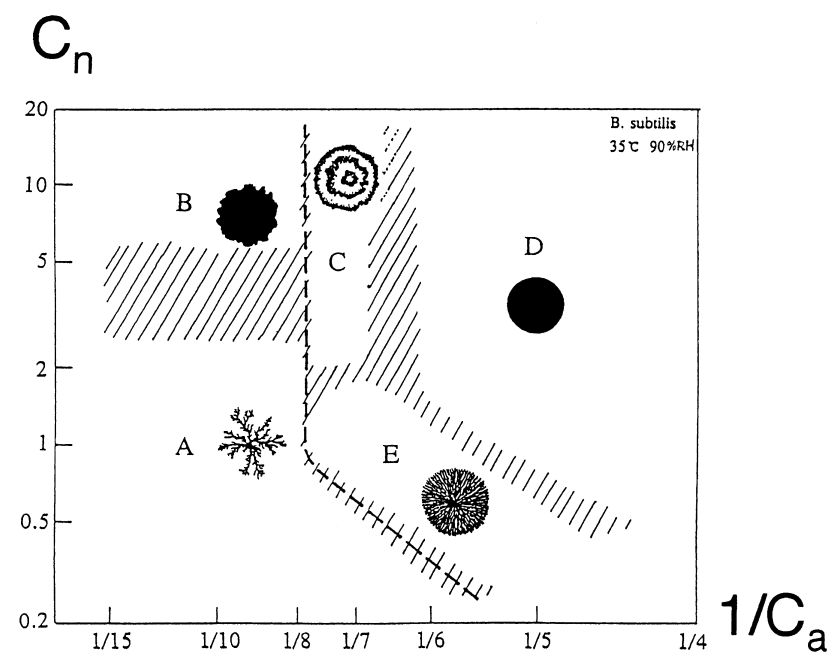

Figure 6. Phase diagram of colonial patterns [12]

between the experimental results and mathematical ones on consumer-finite resource RD systems?" One easy answer is probably to say that the experiment above is not described by an RD system of the form (2.1). In order to judge whether or not this answer is right, we propose some models of bacterial growth in the experiment. Let $b(t, x)$ be the density of bacterial cells at time $t$ and position $\mathrm{x}$. The first equation is

$$
b_{t}=d_{b} \Delta b+\left(b_{0}-\mu b\right)
$$

where $d_{b}$ is the diffusion coefficient of bacterial cells, $b_{0}$ is the initial concentration of nutrients, and $\mu$ is the intra-specific competition rate of cells. These parameters are all positive constants. If the initial function $b(0, x)$ is a pointmass distribution which corresponds to one point inoculation in experiments, then the solution $b(t, x)$ spreads like an expanding disk. In comparison with colony patterns in the region $\mathrm{D}$, this equation is an adequate model to describe patterns observed in the region $\mathrm{D}$ [13]. However, we found that other patterns can never be generated, even if the parameters $d_{b}$ and $n_{0}$ are globally varied. Therefore, we should say that the equation (3.1) is insufficient to answer the above question. As a natural extension of (3.1), the following RD system can 


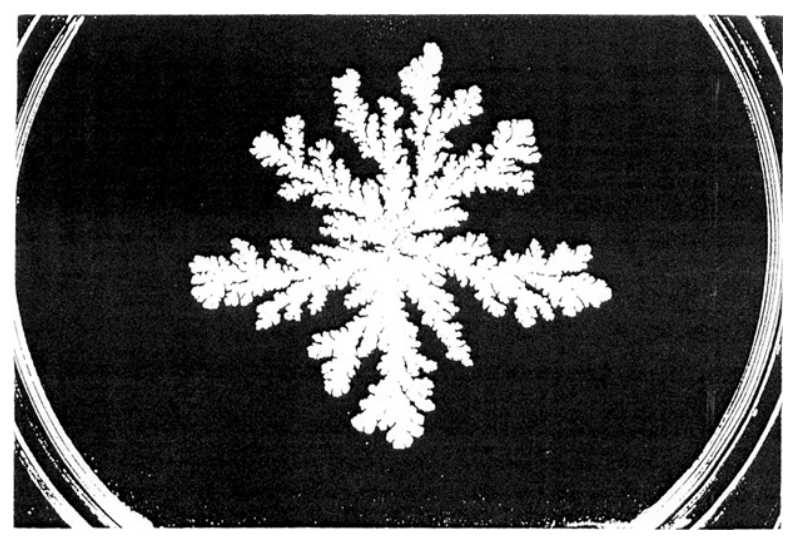

Figure 7. DLA like pattern [12]

be proposed to describe the interaction of bacteria and limited nutrients:

$$
\left\{\begin{array}{l}
b_{t}=d_{b} \Delta b+\omega g(n) b, \\
n_{t}=d_{n} \Delta n-g(n) b,
\end{array}\right.
$$

where $n$ is the concentration of nutrients. $d_{n}$ is the diffusion coefficient of the nutrients and $g(n)$ is the growth rate. A simple form of $g(u)$ is the Malthusian rate $g(n)=\gamma n$ with a positive constant $\gamma . \omega$ is the conversion rate of nutrients from intake to growth. The experiment requires the following initial conditions for $(3.2)$ :

$$
\left\{\begin{array}{l}
b(0, x)=b_{0}(x), \\
n(0, x)=n_{0},
\end{array}\right.
$$

where $b_{0}(x)$ is a point-mass distribution of the initial density of the bacteria and $n_{0}$ is the initial concentration of nutrients which is distributed uniformly in space. We note that the parameters $\left(n_{0}, d_{b} / d_{n}\right)$ in the model $(3.2),(3.3)$ qualitatively correspond to the ones $\left(\mathrm{C}_{\mathrm{n}}, 1 / \mathrm{C}_{\mathrm{a}}\right)$ in the experiment. However, it is numerically well known that the model (3.2) generates disk-like patterns only, even if two parameters are globally varied. As the model is still insufficient, we have to propose a new model. The feature of our model is to introduce the internal state into bacterial cells. That is, if this state variable is relatively large, then the bacteria actively move, grow and perform cell-division, while if it is relatively small, then they do nothing at all. In order to model this in a simple way, we assume that the bacterial cells consist of two types; active cells 


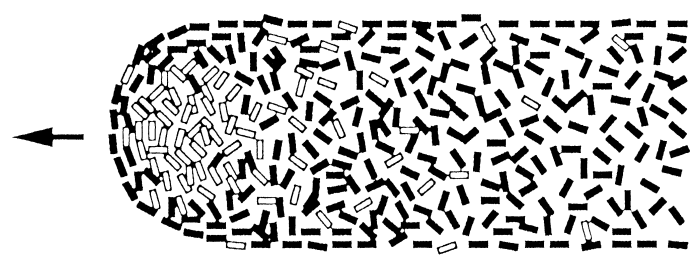

(a)

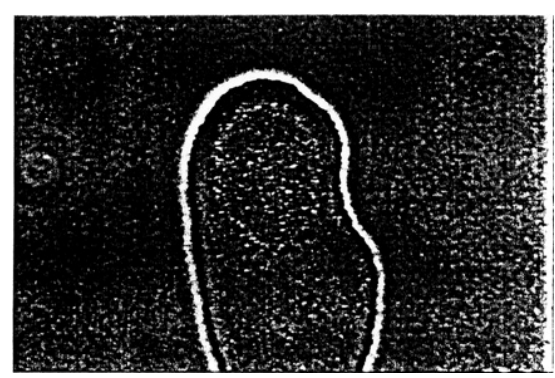

(b)

Figure 8. Finger nail pattern (a) conceptual picture [16]: (b) experiment [12]

and inactive ones. In fact, a group of actively moving cells are clearly observed in the tip of each growing finger in the region E, as if they exhibit finger nail patterns in Figure 8 [12]. They seem to drive the growth of the finger-tip, leaving inactive cells behind.

Let $b(t, x)$ and $s(t, x)$ be the densities of the active and inactive cells at time $t$ and position $x$, respectively, (hence the sum of $b(t, x)+s(t, x)$ is the density of all bacterial cells) and $n(t, x)$ be the concentration of nutrients. The model for $b, s$ and $n$ is given by

$$
\left\{\begin{array}{l}
b_{t}=d_{b} \Delta b+\omega g(n) b-a(b, n) b \\
n_{t}=d_{n} \Delta n-g(n) b \\
s_{t}=a(b, n) b
\end{array}\right.
$$

where $d_{b}$ and $d_{n}$ are the diffusion rates of the bacterial cells and the nutrients, respectively [14]. The growth rate of active cells is $\omega g(n)$. We simply assume $g(n)$ to be the Malthusian growth rate. $a(b, n)$ is the conversion rate from the 
active cells to the inactive ones, which is specified by the mechanism of the internal state. We neglect the conversion rate from the inactive cells to the active ones, because it is observed that once active cells become inactive ones, they never become active again unless food is added artificially. It is plausible that the conversion rate $a(., n)$ decreases as the concentration of nutrients increases. But, the dependence of $a(b,$.$) on the active cells \mathrm{b}$ is still unclear. However, it is observed that if the cell density becomes quite small, each cell is not very active. Thus, we assume that $a(b, n)$ is a decreasing function of $b$ as well as $n$ and that $a(0,0)>0$. For concreteness and simplicity in numerical computation, we adopt the functional form $a(b, n)=a_{0} /\left[1+a_{1} b\right]\left(1+a_{2} n\right)$ with positive constants $a_{i}(i=0,1,2)$. It should be stressed, however, that this particular form is not essential to our conclusion below, and other functions which decrease with $b$ and $n$ work as well.

We simply rewrite (3.4) as the following non-dimensionalized form:

$$
\left\{\begin{array}{l}
b_{t}=d \Delta b+n b-a(b, n) b, \\
n_{t}=\Delta n-n b, \\
s_{t}=a(b, n) b,
\end{array}\right.
$$

where $d$ is the ratio of the diffusion rates $d_{b}$ and $d_{n}$. We consider (3.5) in a bounded domain $\Omega$ with the zero-flux boundary conditions. The initial conditions are

$$
\left\{\begin{array}{l}
b(0, x)=b_{0}(x), \\
n(0, x)=n_{0} \\
s(0, x)=0 .
\end{array}\right.
$$

The first two equations of (3.5) are closed in two unknown variables $b$ and $n$

$$
\left\{\begin{array}{l}
b_{t}=d \Delta b+n b-a(b, n) b, \\
n_{t}=\Delta n-n b .
\end{array}\right.
$$

Then it turns out that (3.7) is a form similar to a class of consumer-finite resource RD system $(2.1)$ except that $a(b, n)$ is not a constant but a function of $b$ and $n$. Since the third variable $s(t, x)$ can be directly obtained in terms of the knowns $b$ and $n$ from (3.7), we can say that (3.5) is essentially two component system for $b$ and $n$. When $a=0$ and $g(n)=n,(3.7)$ obviously reduces to (3.2) so that RD system (3.5) (and also (3.7)) is a slightly modified version of (3.2).

We start with showing the result on asymptotic behavior of solutions to $(3.7)$ : 
Theorem 3 [15]. Let $(b(t, x), n(t, x))$ be a solution of (3.7) in a bounded domain $\Omega$ under the zero flux boundary conditions and the initial conditions (3.6). Then there is some constant $n_{\infty}>0$ such that

$$
\lim _{t \rightarrow \infty}(b(t, x), n(t, x))=\left(0, n_{\infty}\right) .
$$

The result of this theorem is similar to (ii) of Theorem 2, that is, any solution becomes spatially homogeneous asymptotically. (3.7) also falls into the framework of consumer-finite resource RD systems. Biologically speaking, active cells fade out and do not form any pattern. Therefore, we may conclude that RD system (3.7) or (3.5) is less interesting. However, one should not hurry to come to the conclusion, because the bacterial cells are given not by $b(t, x)$ but by $b(t, x)+s(t, x)$. Therefore, we have to know the behavior of $s(t, x)$. For this problem, we have the following result: there is some function $s_{\infty}(x) \geq 0$ such that

$$
\lim _{t \rightarrow \infty} s(t, x)=s_{\infty}(x)
$$

Then, we have

$$
\lim _{t \rightarrow \infty}(b(t, x)+s(t, x))=s_{\infty}(x) .
$$

This result shows that the final colonial pattern of bacterial cells is represented by $s_{\infty}(x)$. Therefore, we need to know the spatial distribution of $s_{\infty}(x)$ which exhibits colonial patterns of bacterial cells and in particular, the dependence of $s_{\infty}(x)$ on values of the parameters $\mathrm{d}$ and $\mathrm{n}_{0}$. Unfortunately, no satisfactory analytic method has not been developed to solve (3.5), (3.6) and therefore we have to rely on numerical methods.

Now, let us numerically consider this problem in a circular domain, taking $n_{0}$ and $d$ as free parameters. Here we only discuss one typical case where $n_{0}$ and $d$ are both small, which corresponds to poor environment (region A). It is shown that one point-mass distribution of $b_{0}(x)$ expands for a short time and then breaks up into several spots. As time goes on, each spot splits into two smaller ones repeatedly so that there appear many spots but they eventually fade out completely. This observation does not contradict the result of Theorem 3 . The behavior of the solution $b(t, x)$ is not so interesting. However, the behavior of $s(t, x)$ is totally different. It exhibits a very complex pattern, as shown in Figure 9(a). A typical feature of the pattern in $s(t, x)$ is the occurrence of tip splitting which corresponds to the situation where a single spot of $b$ splits into two spots. When $n_{0}$ and $d$ decrease, the total bacterial cells $b(t, x)+s(t, x)$ 


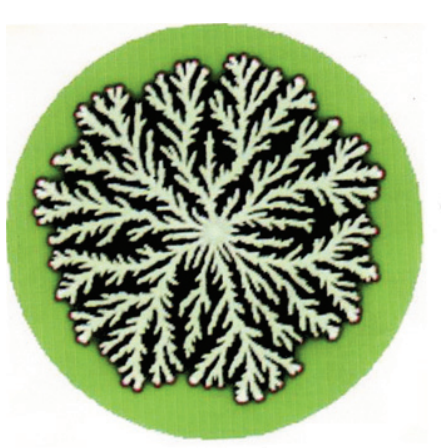

(a)

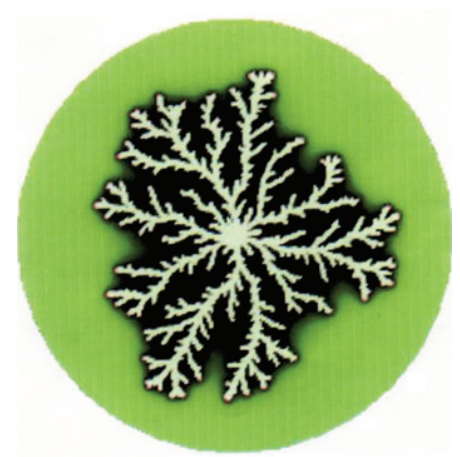

(b)

Figure 9. Numerical branched pattern of $b+s$ in (3.5). (a) DBM-like pattern; (b) DLA-like pattern

exhibit branched patterns similar to DLA-like colonies observed in the region A, as in Figure 9(b). The fractal dimension is about 1.67. On the contrary, for large $n_{0}$ and $d$ (rich environment), $b(t, x)$ exhibits an expanding ring pattern so that the pattern of $b(t, x)+s(t, x)$ simply forms a disk pattern which is similar to the ones in region D. It is surprising that the model (3.5), (3.6) reproduces qualitatively different patterns, depending on values of $n_{0}$ and $d$, despite that the model (3.5) is a consumer-finite resource RD system.

Summarizing the results on our consumer-finite RD model, we can conclude that there is no contradiction between mathematical and experimental results. The asymptotic behavior of active cells $b$ is quite simple but their time history $s$ leave very complex spatio-temporal patterns. 
(a)

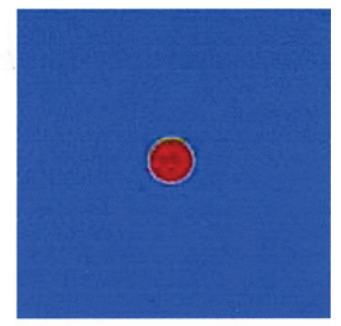

(b)

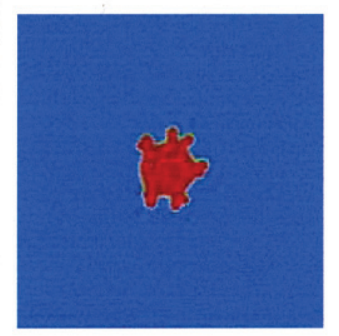

(c)

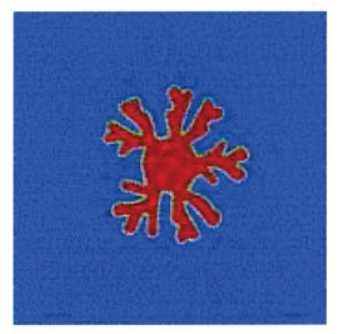

(d)

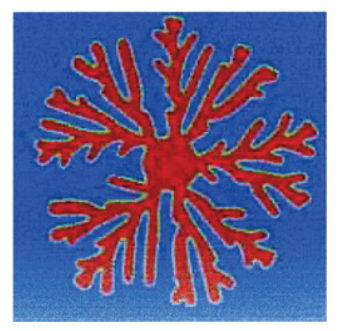

Figure 10. Numerical DLA pattern of $\mathrm{p}$ in (3.8) 
This phenomenon can be also confirmed in the autocatalytic reaction system (2.3). Let us consider the case when $n=2$, and $m=1$,

$$
\left\{\begin{array}{l}
u_{t}=d_{u} \Delta u+k u^{2} v-a u \\
v_{t}=d_{v} \Delta v-k u^{2} v \\
p_{t}=a u
\end{array}\right.
$$

We find that $u, v$ and $p$ correspond to the active cells, nutrients and inactive cells in (3.5), respectively. We already know the asymptotic behavior of $u$ from Theorem 2(i). It fades out asymptotically and when $d_{u} / d_{v}$ is suitably small, the final product $\mathrm{p}$ forms a branched pattern, as in Figure 10, which is qualitatively similar to the pattern in Figure 9(a).

\section{$\S 4$. Conclusion}

We have discussed consumer-finite resource RD systems. It is known that the asymptotic behavior of these systems is qualitatively similar to the corresponding ODEs. For this reason, it had been concluded that these RD systems were simple and abandoned from pattern formation and mathematical analysis viewpoints. However, in this paper, we have shown that this is certainly incorrect, and that depending on the parameters in the system, the time histories of solutions exhibit very complex patterns. No satisfactory analytical method, however, has been developed so far. This should be a future work for us.

\section{Acknowledgements}

The author would like to thank Junko Shigemitu, Mitsugu Matsushita, Hideo Sakaguchi and Daishin Ueyama for their fruitful discussion with him, and also thank an anonymous referee for carefully reading the entire manuscript and for may valuable comments and suggestions.

\section{References}

[1] Turing, A., The basis of morphogenesis, Phil. Trans. Royal Soc. Lond, B237 (1952), 37-72.

[2] Hodgkin, A. L. and Huxley, A. F., A qualitative description of membrane current and its application to conductor and excitation in nerve, J. Physiology, 117 (1952), 500-544.

[3] FitzHugh, R., Impulses and physiological states in theoretical models of nerve membrane, Biophysical J., 9 (1961), 445-466.

[4] Casten, R. G. and Holland, C. J., Instability results for reaction-diffusion equations with Neumann boundary conditions, J. Differential Equations, 27 (1978), 266-273. 
[5] Ikeda, H., Mimura, M. and Nishiura, Y., Global bifurcation phenomena of traveling wave solutions for some bistable reaction-diffusion systems, Nonlinear Anal., 13 (1989), 507-526.

[6] Nishiura, Y., Mimura, M., Ikeda, H. and Fujii, H., Singular limit analysis of stability of travelling wave solutions in bistable reaction-diffusion systems, SIAM J. Math. Anal., 21 (1990), 85-122.

[7] Kermack, W. O. and McKendrick, A. G., A construction to the mathematical theory of epidemics, Part I, Proc. Royal Soc. Lond. Ser. A, 115 (1927), 700-721.

[8] Grey, P. and Scott, S. K., Autocatalytic reactions in the isothermal, continuous stirred tank reactor: Isolas and other forms of multistability, Chem. Eng., 9 (1996), 761-778.

[9] Mimura, M. and Nagayama, M., Non-annihilation dynamics in an exothermic reactiondiffusion system with mono-stable excitability, CHAOS, 7 (1997), 817-826.

[10] Masuda, K., On the global existence and asymptotic behavior of solutions of reactiondiffusion equations, Hokkaido Math. J., 12 (1983), 360-370.

[11] Hoshino, H., On the convergence properties of global solutions for some reaction-diffusion systems under Neumann Boundary conditions, Diff. Integral Eqs., 9 (1996), 761-778.

[12] Matsushita, M. et al., Formation of colony patterns by a bacterial cell population, Physica $A, \mathbf{2 7 4}$ (1999), 190-199.

[13] Wakita, J. et al., Experimental investigation on the validity of population dynamics approach to bacterial colony formation, J. Phys. Soc. Japan, 63 (1994), 1205-1211.

[14] Mimura, M., Sakaguchi, H. and Matsushita, M., Reaction-diffusion modeling of bacterial colony patterns, Phys. A, 282 (2000), 283-303.

[15] Feiresl, E., Hilhorst, D., Mimura, M. and Weidenfeld, R., On nonlinear diffusion system with resource-consumer interaction, Hiroshima Math. J., 33 (2003), 253-295.

[16] Matsuyama, T. and Matsushita, M., Population morphogenesis by cooperative bacteria, FORMA, 16 (2001), 307-326. 